\title{
Impacto de la orientación al aprendizaje en la innovación de las MIPYMES colombianas
}

Impact of learning orientation on innovation in Colombian SMEs

Impact de l'orientation vers l'apprentissage dans l'innovation des PME colombiennes

Fred D. Contreras Palacios, MSc fred.contreras.p@correounivalle.edu.co Universidad del Valle, Cali - Colombia

Magister en Ciencias de la Organización. Profesor Facultad de Ciencias de la Administración Universidad del Valle.

Edgar J. Gálvez Albarracín, PhD edgar.galvez@correounivalle.edu.co Universidad del Valle, Cali - Colombia

Doctor en Administración y Dirección de Empresas. Profesor Facultad de Ciencias de la Administración Universidad del Valle.

Carlos H. González-Campo, PhD carlosh.gonzalez@correounivalle.edu.co Universidad del Valle, Cali - Colombia

Doctor en Administración. Profesor Facultad de Ciencias de la Administración Universidad del Valle.

Artículo de investigación científica y tecnológica Según Clasificación Colciencias

Fecha de recepción: $18 / 08 / 2012$

Fecha de corrección: $15 / 11 / 2012$

Fecha de aprobación: 20/12/2012

\section{Resumen}

Múltiples estudios demuestran que a nivel mundial, la innovación es fuente de ventaja competitiva y que por ello es importante conocer los factores que la estimulan. Es por esto que la investigación que aquí se presenta tiene por objetivo analizar el efecto que tiene la orientación al aprendizaje organizacional sobre la innovación de las empresas. Se realizó un estudio empírico con 403 MIPYMES colombianas. Los resultados obtenidos a través de regresiones lineales múltiples, muestran un efecto altamente positivo en la relación estudiada, lo que tiene importantes implicaciones para empresarios, la academia y el sector gubernamental, porque les indica que un estilo gerencial que estimulen la organización práctica como la divergencia, la generación de ideas novedosas, la toma de riesgos, el intercambio de conocimientos, los acuerdos de cooperación con universidades y otras empresas, la apertura, la experimentación y el cambio continuo, generan un mucho mejor desempeño innovador.

Palabras clave: aprendizaje organizacional, orientación al aprendizaje, innovación, MIPYMES.

Código JEL: D83. 


\section{Impact of learning orientation on innovation in Colombian SMES}

Impacto de la orientación al aprendizaje en la innovación de las MIPYMES colombianas

Impact de l'orientation vers l'apprentissage dans l'innovation des PME colombiennes
Impact de l'orientation vers l'apprentissage dans l'innovation des PME colombiennes

Impacto de la orientación al aprendizaje en la innovación de las MIPYMES colombianas

Impact of learning orientation on innovation in Colombian SMEs

\section{Abstract}

Multiple studies worldwide show that innovation is a source of competitive advantage and it is, therefore, important to know the factors that stimulate it. That is why the research presented here seeks to analyze the effect of organizational learning orientation on business innovation. This empirical study was conducted with 403 Colombian SMEs. The results obtained through multiple linear regression showed a highly positive effect on the relationship studied, which has important implications for employers, academia, and government, as told to a management style that encourages such practices in the organization divergence, the generation of new ideas, risk taking, knowledge sharing, cooperation agreements with universities and other companies, openness, experimentation and continuous change generate better innovation performance.

Keywords: organizational learning, learning orientation, innovation, SME.

Código JEL: D83.

\section{Résumée}

Multiples études démontrent qưà niveau mondial, l'innovation est une source d'avantage compétitif et donc c'est important de connaître les facteurs qui la stimulent. C'est pour ça que cette recherche a pour objectif d'analyser l'effet de l'orientation vers l'apprentissage organisationnel sur l'innovation des entreprises. On a réalisé une étude empirique avec 403 PME colombiennes. Les résultats obtenus par le biais de régressions linéales multiples, montrent un effet hautement positif dans la relation étudiée. Ceci constitue d'importantes implications pour les entrepreneurs, l'académie et le secteur gouvernemental, parce qu'il leur indique qu'un style de direction qui stimule loorganisation pratique, tel que la divergence, la génération de nouvelles idées, la prise de risques, l'échange de connaissances, les accords de coopération avec des universités et d'autres entreprises, l'aperture, l'expérimentation et le changement continu, favorise une meilleure performance innovatrice.

Mots clef: apprentissage organisationnel, orientation vers l'apprentissage, innovation, PME. 


\section{Impacto de la orientación al aprendizaje en la innovación de las MIPYMES colombianas}

\section{Introducción}

Una fuente fundamental para lograr la competitividad de las empresas, reside en sus propios recursos y capacidades, siempre que se adecúen a las exigencias del entorno (Munuera y Rodríguez, 2007; Grant, 1991; Barney, 1991). Los recursos son los activos disponibles y controlables por la empresa, tanto físicos como tecnológicos, humanos y organizativos, mientras las capacidades implican la forma en que los recursos son utilizados por ella.

En este sentido Slater (1996) considera a la innovación y el aprendizaje organizacional como capacidades difíciles de imitar, además son destacados por su potencial para generar otras capacidades (Munuera et al., 2007).

Por su parte, la innovación se considera factor crítico para la supervivencia y el éxito de las empresas (Formichella, 2005; Damanpour y Gopalakrishnan, 2001; Camelo, Romero y Valle, 2000; Rogers, 1983; Schumpeter, 1935), porque las empresas innovadoras son más flexibles y en gran medida, es lo que les permite aprender y adaptarse mejor a los cambios en el entorno, responder más rápido y mejor a las necesidades cambiantes de la sociedad en su conjunto (Drucker, 1985; Miles y Snow, 1978), y obtener así mejores resultados que las que no lo son.

La diferencia entre sobrevivir o no en el actual entorno cambiante de los negocios, tiene que ver con saber más y conocer más rápido que la competencia; en este sentido el aprendizaje organizacional es reconocido como una función organizativa fundamental (Huber, 1998).

La orientación al aprendizaje como uno de los factores que permite el aprendizaje organizacional, se refiere a la actitud y compromiso de los directivos, para incentivar a los empleados a generar nuevas ideas y conocimientos, con una actitud de apertura a la experimentación y para desaprender lo aprendido (Cardona y Calderón, 2006; Calantone, Cavusgil y Zhao, 2002; Baker y Sinkula, 1999), lo cual se considera una inversión a largo plazo que se puede convertir en un factor de supervivencia (Calantone et al., 2002). Con respecto al aprendizaje organizacional y, en particular con la orientación al aprendizaje, se puede percibir en la literatura un creciente interés (Slater y Narver, 1994; Huber 1991; Senge, 1990).

Estudios empíricos previos indican que el aprendizaje organizacional y la innovación constituyen capacidades distintivas y generadoras potenciales de ventaja competitiva en la empresa (Lado y Wilson, 1994), mientras que otros precisan que el aprendizaje y el conocimiento organizacional antecedan a la innovación (Hage, 1999; Nonaka y Takeuchi, 1995), es decir, que para innovar es necesario aprender.

Es importante mencionar que a pesar de lo señalado, en la literatura mundial existen trabajos que analizan la relación entre aprendizaje organizacional e innovación, que están enfocados sobre todo a grandes empresas y abordan poco a las Micro, Pequeñas y Medianas Empresas (MIPYMES); por otra parte, este tipo de estudios son aún escasos en Colombia, a pesar de que según el Departamento Nacional de Estadística (DANE, 2005) en este país ese tipo de empresas constituye el $99.9 \%$ (96.4\% micros, $3.5 \%$ PYMES), generando $63 \%$ del empleo y $37 \%$ de la producción.

Buscando contribuir a llenar el vacío señalado, este trabajo tiene como objetivo analizar empíricamente el impacto de la orientación al aprendizaje sobre la innovación de las MIPYMES. Para esto se realizó un estudio con 403 MIPYMES colombianas, pretendiendo que los resultados permitan al sector gubernamental y la academia diseñar estrategias para mejorar las prácticas de aprendizaje organizacional y de innovación dentro de este tipo de organizaciones.

\section{Marco teórico-conceptual y estudios empíricos previos}

\subsection{Teoría de recursos y capacidades}

En la década de 1990 los investigadores, vuelven a centrar su atención en factores internos de la organización para explicar sus resultados. Así surge, en el seno de la dirección estratégica, la teoría de recursos y capacidades que pretende dar cuenta de estos aspectos internos como 
generadores de ventaja competitiva para la empresa. Para ello, ésta define a la empresa como una colección única de recursos y capacidades (Conner, 1991; Rumelt, 1987; Wernerfelt, 1984), siendo su misión fundamental el estudio de las diferencias en los resultados empresariales y su premisa principal la presencia de heterogeneidad entre las empresas en cuanto a los recursos que controlan, convirtiéndose ésta en el principal factor que explica la diferencia entre los diversos resultados obtenidos por cada una de ellas (Barney, 1991; Rumelt, 1984; Lippman y Rumelt, 1982).

Desde esta perspectiva, las diferencias entre los niveles de éxito alcanzados por las empresas se explican, más por los recursos y capacidades que por las características estructurales del sector al que pertenece la empresa (Barney, 1991). En esta dirección Day (1993, p. 95) afirma que "El vínculo entre las capacidades de la organización y los retos a los que se enfrenta son el elemento más importante a la hora de comprender el éxito y el fracaso de las empresas".

En este sentido, una fuente vital para lograr la competitividad de las empresas, reside en sus propios recursos y capacidades, siendo los recursos los activos disponibles y controlables por la empresa, tanto físicos como tecnológicos, humanos y organizativos, mientras las capacidades implican la forma en que los recursos son utilizados por ella (Barney, 1991).

\subsection{Aprendizaje organizacional}

La teoría moderna de la organización sostiene la idea de que las organizaciones aprenden, porque modifican sus metas y procedimientos cotidianos teniendo en cuenta sus experiencias (Cyert y March, 1963). Drucker (1993, p. 1) afirmaba que "cada pocos centenares de años ocurre en la historia del occidente una notable transformación", indicando que hemos pasado de hablar de "sociedad de empleados" y de una "sociedad de organizaciones" a hablar de una "sociedad del conocimiento" y del "trabajador de conocimiento". El recurso económico ya no será el capital ni los recursos naturales, es y será el conocimiento (Drucker, 1993).

Tratando de construir una definición a partir de distintos autores, Garzón y Fischer (2009, p. 249) definen el aprendizaje organizacional como:
La capacidad de las organizaciones de crear, organizar y procesar información desde sus fuentes, para generar nuevo conocimiento individual, de equipo, organizacional e inter-organizacional, generando una cultura que lo facilite y permitiendo las condiciones para desarrollar nuevas capacidades, diseñar nuevos productos y servicios, incrementar la oferta existente y mejorar procesos orientados con la perdurabilidad".

La orientación al aprendizaje se ha considerado como una dimensión del aprendizaje organizacional (Calantone et al., 2002; Baker y Sinkula, 1999). Es así como Cardona y Calderón (2006) identifican tres dimensiones que permiten el aprendizaje organizacional: el conocimiento compartido, la recuperación del conocimiento y la orientación del aprendizaje.

\subsection{Orientación al aprendizaje organizacional}

La orientación al aprendizaje se puede conceptualizar como un conjunto de valores organizacionales que influyen tanto en la empresa para crear y utilizar el conocimiento (Sinkula et al., 1997), como el grado en que se satisface la empresa con sus paradigmas (Argyris y Schon, 1978), modelos mentales (Geus, 1988), y con las lógicas dominantes (Bettis y Prahalad, 1995). En este sentido se han asociado habitualmente tres valores organizacionales que predisponen a las organizaciones a aprender, estos son: el compromiso con el aprendizaje, una mente abierta, y una visión compartida (Day, 1994, 1991; Senge, 1990).

De otra parte Weerawardena, $0^{\prime}$ Cass y Julian (2006) diferencian tres tipos de orientación al aprendizaje: la orientación al aprendizaje de mercado, la orientación al aprendizaje relacional y la orientación al aprendizaje interno. De este último, algunos autores solo reconocen tres dimensiones compromiso con el aprendizaje, mentalidad abierta y visión compartida (Sinkula et al., 1997; Day, 1994, 1991; Senge, 1990), en tanto otros lo conciben con cuatro factores: compromiso con el aprendizaje, mentalidad abierta, visión compartida y el intercambio de conocimiento (Hurley y Hult, 1998).

Desde la orientación del aprendizaje propuesta en Cardona y Calderón (2006), se definen tres dimensiones: el compromiso con 
el aprendizaje, la capacidad para desaprender lo aprendido, y la apertura y experimentación. A continuación presentamos cada uno de ellos.

\section{Compromiso con el aprendizaje}

El compromiso con el aprendizaje es el grado en que una organización valora y promueve el aprendizaje (Sinkula et al., 1997), considerando éste como una inversión a largo plazo que se convierte en un factor de supervivencia (Calantone et al., 2002). Este compromiso y esta capacidad no implican, como lo expresa Senge (2000), forzar, apresurar ni imponer.

De tal manera, Paños, Ruiz, Sabater y Ruiz (2004) plantea que para fomentar la generación de nuevo conocimiento a nivel individual es necesario, entre otros aspectos, que las organizaciones fomenten la autonomía, la creatividad, la motivación y la superación y en algunos casos exigen a los empleados a cuestionar constantemente las normas que guían las acciones de la organización (Sinkula et al., 1997; Day, 1991).

\section{Desaprendizaje}

Tradicionalmente las empresas y las personas se esfuerzan en aprender; sin embargo, uno de los mayores obstáculos para producir nuevo conocimiento es el desaprender lo que sabe, entendido esto último como el proceso mediante el cual se desecha el conocimiento obsoleto y engañoso (Cegarra y Rodrigo, 2004).

De otra parte Huber (1991) manifiesta que uno de los principales factores que influyen en el éxito del aprendizaje organizacional es la eliminación del conocimiento obsoleto. Esto solo sucede si en la organización se dan las condiciones que lo fomenten; para este fin requiere ser autorizado por la dirección y no ser obstruido por reglas o regulaciones (Cegarra y Rodrigo, 2004).

Por consiguiente, un factor importante para que el aprendizaje organizativo se geste en la organización consiste en eliminar los modelos mentales implícitos en la estructura de la empresa, que aun cuando fueron exitosos en el pasado en la actualidad afectan negativamente las reglas que determinan el comportamiento de individuos y grupos en la organización (Grant, 1991). De esta manera están promoviendo el desaprendizaje cuando las organizacio- nes de forma proactiva cuestionan sus rutinas y creencias (Baker y Sinkula, 1999).

\section{Apertura y experimentación}

Weerawardena et al., (2006) concibe la orientación al aprendizaje interno como la capacidad de una empresa para desarrollar conocimiento a través de sus recursos internos, jugando así la investigación y desarrollo un papel decisivo. Este aprendizaje disemina, desecha o usa el conocimiento para la realización de cambios en la organización. Al respecto Dixon (1992) y Huber (1991) consideran que el aprendizaje enfocado al interior incluye el aprendizaje experiencial (aprendizaje por ensayo y error), y el aprendizaje experimental (desarrollo de nuevas formas de hacer las cosas).

En este sentido, conforme pasa el tiempo, los modelos mentales tradicionales se vuelven obsoletos ante los cambios suscitados en el entorno (Baker y Sinkula, 1999), limitando a la organización a las formas habituales de pensar y actuar. Esto puede ocurrir si no existe en la organización una mente lo suficientemente abierta para cuestionar estas formas de pensar y actuar (Porac y Thomas, 1990; Senge, 1990). Cuando esto último ocurre, se puede plantear que la apertura mental está ligada al desaprendizaje (Nystrom y Starbuck, 1984).

\subsection{Innovación}

El Manual de Oslo (OECD y EUROSTAT, 2005) señala que la innovación es "la introducción de un nuevo o significativamente mejorado producto (bien o servicio), de un proceso, de un nuevo método de comercialización o de un nuevo método organizativo, en las prácticas internas de la empresa, la organización del lugar de trabajo o las relaciones exteriores".

En este sentido, la innovación es el proceso de integración de la tecnología existente y los inventos para crear o mejorar un producto, un proceso o un sistema. Innovación, en un sentido económico, consiste en la consolidación de un nuevo producto, proceso o sistema mejorado (Medina y Espinosa, 1994). La innovación consiste en producir, asimilar y explotar con éxito la novedad en los ámbitos económico y social (COM, 2003).

De otra parte la Asociación Española de Contabilidad y Administración de Empresas (AECA) 
(1995), señala que en la organización se pueden presentar tres tipos de innovación: de productos, de procesos y de gestión. La innovación en productos se materializa en la comercialización de un nuevo artículo o en la mejora de otro existente, la innovación en procesos dota a la empresa de nuevos equipos o nuevos procesos de producción.

\subsection{Estudios empíricos sobre la relación entre la orientación al aprendizaje y la innovación empresarial}

Estudios empíricos previos indican la existencia de una relación fuerte entre la orientación al aprendizaje y la innovación empresarial (Calantone et al., 2002; Sinkula et al., 1997; Thompson, 1965), entre estos se pueden señalar los siguientes:

Abbas y Alireza (2011) adelantaron un estudio en 82 empresas de Teherán con el propósito de conocer el efecto de la orientación del aprendizaje en la innovación. El estudio mostró que existen relaciones significativamente positivas entre estos.

Calantone et al. (2002) a través de entrevistas en profundidad a 400 vicepresidentes de industrias manufactureras y de servicios en Estados Unidos, realizaron una investigación con el fin de conocer el efecto de la orientación al aprendizaje sobre la innovación. Los resultados evidenciaron que existe una relación positiva entre estas variables.

Ma, Zhu y Hou(2011), utilizaron datos de 534 plantas de producción en la industria manufacturera de la provincia de Guangdong (China), y realizaron un estudio sobre las relaciones entre la orientación al aprendizaje y la innovación de procesos a través de modelos de ecuaciones estructurales. Este estudio demostró que la orientación al aprendizaje tiene un efecto directo y positivo en la innovación de procesos. También encontraron que el proceso de innovación es un mediador en la relación entre la orientación al aprendizaje y resultados de la empresa.

Con otra perspectiva García-Morales, MatíasReche y Verdú-Jover (2011), formularon un modelo global para analizar las relaciones directas e indirectas entre las variables comunicación interna, proactividad tecnológica, aprendizaje organizacional, innovación organizacional y desempeño. Este modelo fue probado en 164 empresas tecnológicas de Europa y de Estados Unidos. Entre otros, los resultados mostraron que existe una asociación positiva y estadísticamente significativa entre el aprendizaje organizacional y la innovación.

Jiménez y Sanz (2006) estudiaron el impacto que el aprendizaje organizacional tiene sobre la innovación en una muestra de 451 empresas españolas pertenecientes a los sectores industrial y de servicios. Encontraron que existen evidencias significativas que apoyan esta relación. El aprendizaje se midió desde la perspectiva de Huber (1991): adquisición de conocimiento, distribución del conocimiento, interpretación y memoria organizativa.

Consecuentemente con el marco teórico y los estudios previos revisados, se plantea la siguiente hipótesis de investigación:

H1: La orientación al aprendizaje influye positivamente en la innovación empresarial.

\section{Metodología de la investigación}

Este trabajo es de tipo correlacional con enfoque cuantitativo, porque tiene como propósito medir empíricamente el grado de relación entre la orientación al aprendizaje y la innovación organizacional. Los datos se obtuvieron de fuentes primarias a través de una encuesta. Para contrastar la hipótesis se realizó un análisis multivariante mediante regresiones lineales múltiples. A continuación se describen los diferentes aspectos de la metodología empleada para la investigación:

\subsection{Obtención de la muestra y recolec- ción de datos}

Las empresas objeto del estudio son $403 \mathrm{MI}$ PYMES formalmente constituidas, pertenecientes a la industria, la construcción, el comercio y los servicios, ubicadas en diferentes ciudades de Colombia. La muestra se obtuvo mediante muestreo estratificado. Dentro de cada estrato la selección se hizo mediante un muestreo aleatorio simple con un error muestral de 4.9 puntos y un nivel de confianza del $95 \%$ (Tablas 1.1 y 1.2).

La recolección de los datos se realizó durante los meses de junio y julio del año 2011, en el marco del proyecto de investigación "Análisis estratégico para el desarrollo de las MIPYMES colombianas", con código interno C.I. 8088 de la Universidad del Valle. El instrumento utilizado fue una encuesta estructurada que se aplicó a los dueños o gerentes de las empresas. 


\begin{tabular}{|c|c|}
\hline Región y ciudades & $\begin{array}{l}\text { Número de } \\
\text { empresas }\end{array}$ \\
\hline Centro (Bogotá, Bucaramanga) & 133 \\
\hline Antioquia (Medellín) & 89 \\
\hline Suroccidente (Cali, Popayán, Pasto) & 87 \\
\hline Norte (Barranquilla, Cartagena, Santa Marta) & 70 \\
\hline Eje cafetero (Armenia, Manizales, Pereira) & 24 \\
\hline Total & 403 \\
\hline
\end{tabular}

Fuente: Contreras, F. D., Gálvez, E. J. y González, C. H. (2012)

\begin{tabular}{|c|c|c|}
\hline \multicolumn{3}{|c|}{ Tabla 1.2. Distribución de la muestra por sectory tamaño } \\
\hline Sector & Muestra & Error (\%) \\
\hline Industria & 198 & 6.9 \\
\hline Construcción & 21 & 21.3 \\
\hline Comercio & 75 & 11.2 \\
\hline Servicios & 109 & 9.4 \\
\hline Tamaño (N trabajadores) & \\
\hline 5 a 10 & 125 & 8.7 \\
\hline 11 a 50 & 196 & 7.0 \\
\hline 51 a 250 & 82 & 10.8 \\
\hline Total & 403 & 4.9 \\
\hline
\end{tabular}

Fuente: Contreras, F. D., Gálvez, E. J. y González, C. H. (2012)

\subsection{Medición de las variables}

\subsubsection{Orientación al aprendizaje organizacional}

En este trabajo, después de la revisión de los instrumentos utilizados en otros estudios empíricos (Goh y Richard, 1997; Sinkula et al., 1997; Nonaka y Takeuch, 1995; Day, 1994) y conforme al interés de la investigación, se adoptó parcialmente la escala desarrollada por Cardona y Calderón (2006) para medir el aprendizaje organizacional, porque una de las dimensiones que considera es la orientación al aprendizaje.

Como la orientación al aprendizaje es una construcción de segundo orden (Ma et al., 2011; Sinkula et al., 1997), para medir la orientación al aprendizaje, Cardona y Calderón (2006) incorporan en su instrumento tres indicadores de primer orden que son las que se utilizaron en este trabajo, a saber: 1) compromiso de directivos y demás empleados (ORIENCOM), 2) apertura y experimentación (ORIENEXP) y 3) desaprendizaje (ORIENDES)

Con los indicadores señalados se construyeron 9 ítems, por medio de los cuales se midió esta variable utilizando una escala Likert de 1 a 5 (donde 1 es total desacuerdo y 5 total acuerdo).

\subsubsection{Variable de innovación}

Para medir el grado de innovación de la empresa existen dos enfoques (Hughes, 2007), un enfoque objetivo que mide a partir de datos de tipo cuantitativo, como número de patentes o datos específicos de la innovación en productos (número de nuevos productos) o procesos (costes de inversión). Y un enfoque subjetivo, basado en la percepción del gerente o propietario de la empresa sobre su actividad innovadora. En el caso de la Pequeña y Mediana Empresa resulta más apropiado el enfoque subjetivo, porque el enfoque objetivo tiende a subestimar la actividad innovadora de las Pyme (Hughes, 2007). Este enfoque, por ejemplo, es el que se utiliza en el Estudio de la Innovación Armonizada de la Unión Europea.

Para analizar este factor en la investigación, se tuvieron en cuenta los diferentes conceptos recopilados en la revisión de trabajos realizados y en especial la de AECA (1995) que señaló que la innovación puede clasificarse en tecnológica y organizacional. Las innovaciones tecnológicas incluyen las novedades significativas en productos y en procesos; la innovación organizacional por su parte corresponde a los cambios introducidos a la estructura administrativa de la empresa, a la comercialización, a la financiación, y demás. Esta misma clasificación se ha usado en diferente trabajos (Maldonado et al., 2009).

Para medir el grado de innovación en: 1) productos (INNOVAPS), 2) procesos (INNOVAPC) y 3) gestión (INNOVAGE), se utilizó una medida multicriterio con dos o más ítems por cada tipo de innovación, con una escala Likert de 5 puntos. La variable que representa la innovación global se construye a partir de la media aritmética de las 3 medidas de innovación, también en una escala teórica de 1 a 5 .

\subsubsection{Modelo analizado}

Para verificar la hipótesis planteada se formuló el siguiente modelo:

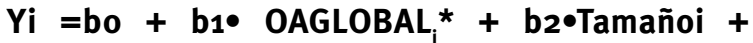 b3•Edadi}

Donde:

Variable dependiente $=Y i$

INNOVAPS Innovación en productos 


\section{INNOVAPC}

INNOVAGE

Innovación en procesos

Innovación en gestión

\section{Variable explicativa:}

\section{OAGLOBAL * Orientación al aprendizaje global}

Variables de control:

Tamaño: Se introduce al modelo como variable ficticia de dos categorías (D. pequeña, D. mediana).

Edad: Se introduce al modelo como variable ficticia de una categoría (D. madura).

En esta investigación, la orientación al aprendizaje es un constructo de segundo orden compuesto por el compromiso con el aprendizaje (ORIENCOM), apertura y experimentación (ORIENEXP) y el desaprendizaje (ORIENDES). En el Gráfico 1 se presenta la relación entre las variables consideradas y la hipótesis planteada.

Grafico 1. Relación entre la orientación al aprendizaje

y la innovación empresarial

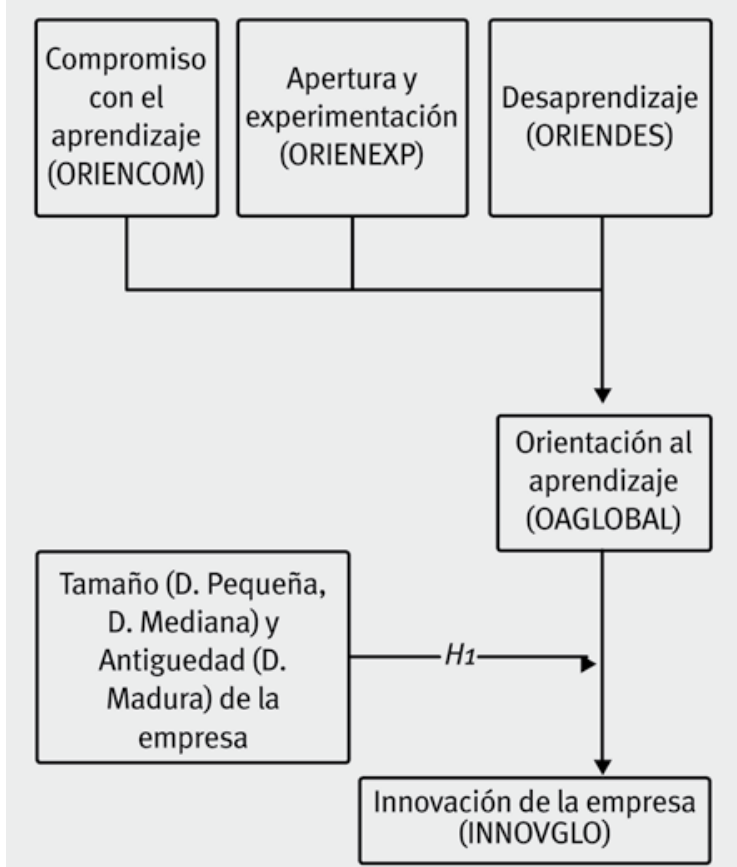

* Productos (INNOVAPS)

* Procesos (INNOVAPC)

* Gestión (INNOVAGE)

Fuente: Contreras, F. D., Gálvez, E. J. y González, C. H. (2012).

\subsection{Método analítico}

Para contrastar las hipótesis se ha utilizado el análisis de regresión lineal múltiple. El modelo analítico exige que se cumplan una serie de supuestos: linealidad del fenómeno, la varianza constante, la independencia y la normalidad de la distribución de los términos de error (Hair, Anderson, Tathamy Black, 1999). Estos han sido comprobados observándose su cumplimiento. Finalmente todos los análisis se han desarrollado de forma separada teniendo en cuenta cada sector: industria, comercio, servicio y construcción.

\section{Análisis de fiabilidad $y$ validez de las escalas}

Con el fin de comprobar el grado de fiabilidad, entendido como consistencia interna, en primer lugar se calculó el coeficiente alfa de Cronbach (a) para cada una de las dimensiones del estudio (Tabla 2). Aun cuando no todas las dimensiones de los constructos superan el umbral recomendado por Nunnally de 0,70 , sí lo logran los constructos orientación al aprendizaje $(a=0,809)$ e innovación $(a=0,849)$. Eso significa que tienen un alto grado de consistencia interna.

\begin{tabular}{|c|c|}
\hline Dimensión & $\begin{array}{c}\text { a de } \\
\text { Cronbach }\end{array}$ \\
\hline $\begin{array}{l}\text { Compromiso de directivos y demás } \\
\text { empleados }\end{array}$ & 0,715 \\
\hline Apertura y experimentación & 0,686 \\
\hline Desaprendizaje & 0,414 \\
\hline Total orientación al aprendizaje & 0,809 \\
\hline Productos/servicios & 0,695 \\
\hline Procesos & 0,665 \\
\hline Sistemas de gestión & 0,737 \\
\hline Total innovación & 0,849 \\
\hline
\end{tabular}

En esta investigación se ha utilizado un análisis factorial de componentes [AFC] con rotación varimax para poner a prueba la unidimensionalidad y la fiabilidad de los ítems que conforman cada constructo. Las cargas factoriales se muestran en la Tabla 3, 
donde se puede observar un buen grado de coherencia entre los ítems para medir cada constructo y que el conjunto de ítems para cada constructo es unidimensional en un solo factor.

\begin{tabular}{|c|c|c|}
\hline Dimensión & Îtem & CARGA \\
\hline \multicolumn{3}{|c|}{ Orientación al aprendizaje } \\
\hline \multirow[t]{4}{*}{$\begin{array}{l}\text { Compromiso con } \\
\text { laprendizaje }\end{array}$} & $\begin{array}{l}\text { 1. Los empleados en esta empresa frecuentemente proponen nuevas ideas e introducen } \\
\text { novedades en su trabajo }\left(\mathrm{P}_{3101}\right)\end{array}$ & ,634 \\
\hline & $\begin{array}{l}\text { 2. Los directivos de esta organización exploran continuamente el ambiente para ganar } \\
\text { nuevas perspectivas }\left(\mathrm{P}_{3102}\right)\end{array}$ & ,751 \\
\hline & $\begin{array}{l}\text { 3. El equipo directivo de nuestra organización, fomenta entre sus colaboradores, la iniciativa, } \\
\text { la innovación, la asunción de riesgos y el intercambio de conocimientos }\left(\mathrm{P}_{31} \mathrm{~B}_{3}\right)\end{array}$ & ,785 \\
\hline & $\begin{array}{l}\text { 4. La dirección impulsa acuerdos de colaboración con universidades y/u otras empresas } \\
\text { para intercambiar conocimientos y experiencias }\left(\mathrm{P}_{3104)}\right.\end{array}$ & ,504 \\
\hline \multirow[t]{3}{*}{$\begin{array}{l}\text { Aperturay } \\
\text { experimentación }\end{array}$} & $\begin{array}{l}\text { 5. En nuestra organización las ideas innovadoras que funcionan son con frecuencia } \\
\text { recompensadas }\left(P_{3105}\right)\end{array}$ & 699 \\
\hline & 6. Las fallas son discutidas constructivamente en nuestra organización (P3106) &, 721 \\
\hline & $\begin{array}{l}\text { 7. Las personas en esta organización son animadas a que cuestionen la forma de hacer las } \\
\text { cosas }\left(\mathrm{P}_{3107)}\right.\end{array}$ & 669 \\
\hline \multirow[t]{2}{*}{ Desaprendizaje } & $\begin{array}{l}\text { 8. Los administradores de nuestra empresa son capaces de romper con las perspectivas } \\
\text { tradicionales para ver las cosas en nuevas y diferentes formas }\left(\mathrm{P}_{3108)}\right.\end{array}$ & ,699 \\
\hline & $\begin{array}{l}\text { 9. Los empleados de nuestra organización resisten el cambio y tienen miedo de nuevas ideas } \\
\text { (P3109) }\end{array}$ & 324 \\
\hline \multicolumn{3}{|c|}{ (1) } \\
\hline \multirow{2}{*}{$\begin{array}{l}\text { Productos/ } \\
\text { servicios }\end{array}$} & 1. Cambios o mejoras en productos/servicios existentes ( $P_{2301 a)}$ & ,697 \\
\hline & 2. Comercialización nuevos productos/servicios (P2302a) & ,748 \\
\hline \multirow[t]{2}{*}{ Procesos } & 3. Cambios o mejoras en los procesos de producción/servicios (Р230за) & ,774 \\
\hline & 4. Adquisición de nuevos bienes de equipos (P2304a) &, 717 \\
\hline \multirow[t]{3}{*}{ Gestión } & 5. Cambios o mejoras en dirección y gestión (P2305a) & ,743 \\
\hline & 6. Cambios o mejoras en compras y aprovisionamientos (P2306a) & ,665 \\
\hline & 7. Cambios o mejoras en comercial/ventas (P2307a) & ,755 \\
\hline
\end{tabular}

Fuente: Contreras, F. D., Gálvez, E. J. y González, C. H. (2012). Método de extracción: análisis de componentes principales. Normalización varimax. Un componente extraído.

Como en esta investigación, la orientación al aprendizaje es un constructo de segundo orden (Sinkula et al., 1997) compuesto por el compromiso de directivos y demás empleados, apertura y experimentación, y por el desaprendizaje (Cardona y Calderón, 2006). Con el fin de confirmar que la orientación al aprendizaje es un factor de segundo orden, también se realizó un análisis factorial de componentes principales (Tabla 4). Se puede observar que, todos los pesos de los factores son superiores a 0.50 , lo que implica que el modelo se ajusta muy bien a los datos (Ma et al., 2011).

Tabla 4. Matriz de componentes principales de la orientación al aprendizaje

\begin{tabular}{|c|c|}
\hline Dimensión & Carga \\
\hline ORIENCOM &, 836 \\
ORIENEXP &, 869 \\
ORIENDES &, 732 \\
\hline
\end{tabular}

Fuente: Contreras, F. D., Gálvez, E. J. y
González, C. H. (2012).

\section{Validez convergente}

Teniendo en cuenta que la validez es el grado en que la medida representa con pre- cisión lo que se supone representa (Hair et al., 1999), ésta existirá cuando se obtenga una correlación significativa (al 1\%), entre las dimensiones que constituyen un constructo (Jerez, 2001). En esta investigación nuestros constructos son: la orientación al aprendizaje y la innovación.

Como se puede observar en la Tabla 5 , existe para la orientación al aprendizaje, una correlación significativa entre las dimensiones compromiso de directivos y demás empleados (ORIENCOM), apertura y experimentación (ORIENEXP), y desaprendizaje (ORIENDES).

\begin{tabular}{l}
$\begin{array}{l}\text { Tabla 5.Correlaciones entre las dimensiones de la } \\
\text { orientación al aprendizaje }\end{array}$ \\
\begin{tabular}{l|c|c|c|}
\hline ORIENCOM & 1 & \\
\hline ORIENEXP & $.626^{\star \star}$ & \\
\hline ORIENDES & $.388^{\star \star}$ & $.461^{\star \star}$ & 1 \\
\hline \multicolumn{3}{|c|}{ Fuente: Contreras, F. D., Gálvez, E. J.y } \\
González, C. H. (2012).
\end{tabular} \\
\hline
\end{tabular}


Finalmente, para el constructo de la innovación (Tabla 6), se observa una correlación significativa al $1 \%$ entre sus dimensiones productos/ servicios (INNOVAPS), procesos (INNOVAPC) y gestión (INNOVAGE).

\begin{tabular}{|c|c|c|c|}
\hline & INNOVAGE & INNOVAPS & INNOVAPC \\
\hline INNOVAGE & 1 & & \\
\hline INNOVAPS & $.552^{\star \star}$ & 1 & \\
\hline INNOVAPC & $.611^{\star \star}$ & $.607^{\star \star}$ & 1 \\
\hline \multicolumn{4}{|c|}{ Correlación significativa al $0,01^{\star \star}$} \\
\hline
\end{tabular}

Teniendo en cuenta los resultados anteriores se confirma la existencia de validez convergente en cada uno de los constructos: la orientación al aprendizaje y la innovación.

\section{Resultados y discusión}

La Tabla 7, muestra los resultados de las estimaciones realizadas, examinando las relaciones entre la orientación al aprendizaje global (OAGLOBAL), la antigüedad de la empresa (representada por la variable ficticia: D. madura), tamaño de la empresa (representada por las variables ficticias: D. pequeña, D. mediana), frente a la innovación global (INNOVGLO) y sus tres dimensiones: productos/servicios (INNOVAPS), procesos (INNOVAPC) y gestión (INNOVAGE). A continuación se describen y discuten los resultados de cada relación:

\section{Efecto de la orientación al aprendizaje en la innovación de productos}

La orientación al aprendizaje global tiene coeficientes positivos y significativos para la innovación en productos $\left(\beta=0,289^{c}\right)$ y con una validez global altamente significativa $\left(\mathrm{F}=7,094^{\mathrm{c}}\right.$. Esto implica que los valores de la organización en lo que respecta a su compromiso con el aprendizaje, la experimentación y el desaprendizaje, promueven mejoras en los productos de la MIPYME y en su comercialización. Esto coincide con los hallazgos de España (Jiménez y Sanz, 2006).

\begin{tabular}{|c|c|c|c|c|}
\hline \multirow{2}{*}{$\begin{array}{l}\text { Variable Dependiente } \\
\text { Variables independientes }\end{array}$} & \multicolumn{4}{|c|}{ Innovación } \\
\hline & Producto / servicio & Procesos & Gestión & Global \\
\hline $\mathbf{R}^{2}$ ajustado & 0.081 & 0.12 & 0.091 & 0.119 \\
\hline Durvin-Watson & 1.698 & 1.810 & 1.972 & 1.831 \\
\hline $\mathbf{F}$ & $7.094^{C}$ & $10.109^{C}$ & $5.957^{C}$ & $5.92^{C}$ \\
\hline VIF más alto & 1.638 & 1.710 & 1.639 & 1.709 \\
\hline$\underset{t}{\text { OAGLOBAL: } \beta}$ & $\begin{array}{l}0.289^{C} \\
4.883\end{array}$ & $\begin{array}{l}0.282^{C} \\
4.734\end{array}$ & $\begin{array}{l}0.330^{C} \\
4.829\end{array}$ & $\begin{array}{c}0.329^{C} \\
4.197\end{array}$ \\
\hline$\underset{t}{\text { D. madura } \beta}$ & $\begin{array}{l}-0.032 \\
-0.530\end{array}$ & $\begin{array}{l}-0.090 \\
-1.480\end{array}$ & $\begin{array}{l}-0.016 \\
-0.224\end{array}$ & $\begin{array}{l}-0.015 \\
-0.185\end{array}$ \\
\hline D. pequeña $\beta$ & $\begin{array}{l}0.078 \\
1.092\end{array}$ & $\begin{array}{l}0.207^{C} \\
2.681\end{array}$ & $\begin{array}{l}0.015 \\
0.179\end{array}$ & $\begin{array}{c}0.185^{\mathrm{a}} \\
1.911\end{array}$ \\
\hline D. mediana $\beta$ & $\begin{array}{l}0 . .053 \\
0.723\end{array}$ & $\begin{array}{l}0.207^{b} \\
2.861\end{array}$ & $\begin{array}{l}0.004 \\
0.049\end{array}$ & $\begin{array}{l}0.129 \\
1.267\end{array}$ \\
\hline
\end{tabular}

Fuente: Contreras, F. D., Gálvez, E. J. y González, C. H. (2012).

\section{Efecto de la orientación al aprendizaje en la innovación de procesos}

La segunda relación analizada explica significativamente la variabilidad positiva de la innovación en procesos a través de la orientación al aprendizaje global porque el coeficiente es significativo y positivo $\left(\beta=0,282^{c}\right)$, y tiene una fuerza de asociación superior al de productos $\left(F=10,109^{c}\right)$. Estos resultados dan cuenta de que la disposición al aprendizaje organizacional genera cambios o mejoras de los procesos de producción o de servicios, al igual que en la adquisición de nuevos equipos. Estos hallazgos coinciden con los obtenidos por Ma et al., (2011). Es importante también señalar en esta relación, que el tamaño 
de la empresa influye significativamente en la innovación de procesos, siendo superior en las pequeñas y medianas empresas.

\section{Efecto de la orientación al aprendizaje en la inno- vación en gestión}

La innovación en gestión se ve también positivamente afectada por la orientación global al aprendizaje porque muestra un coeficiente positivo y significativo $\left(\beta=0,330^{\circ}\right)$ y una validez global altamente significativa $\left(F=5,957^{c}\right)$. Esto indica que el compromiso con el aprendizaje, la apertura, la experimentación y el desaprendizaje, generan en los directivos de las MIPYME, mejoras en su forma de dirigir, comercializar o comprar. Al igual que en la innovación en productos no se encontraron estadísticos significativos en el tamaño y la edad de las empresas; esto quiere decir que estos dos factores no influyen en esta relación.

\section{Efecto de la orientación al aprendizaje en la innovación global}

Finalmente, la orientación al aprendizaje muestra un efecto favorable sobre la innovación global, porque el estadístico correspondiente es significativo y positivo $\left(\beta=0,329^{c}\right)$; y la varianza también $\left(F=5.920^{c}\right)$. Esto quiere decir que la sumatoria de las diferentes prácticas asociadas con la orientación al aprendizaje organizacional, influye en el desempeño innovador general de la MIPYME. Estos resultados coinciden de los que obtuvieron Abbas y Alireza (2011) y Ma et al., (2011), que encontraron una significativa y positiva relación entre la orientación al aprendizaje y la innovación.

Con base en todos los resultados señalados, se puede confirmar la hipótesis contrastada porque para todas las relaciones analizadas se encontraron estadísticos positivos y significativos.

\section{Conclusiones, implicaciones y futuras líneas de investigación}

La importancia de analizar la orientación al aprendizaje organizacional, radica en que está asociado con el desarrollo de nuevo conocimiento, que a su vez es crucial para estimular la capacidad de innovación y la generación de los resultados empresariales en general (Hurley y Hult, 1998; Slater y Narver, 1994). Bajo este contexto, esta investigación tuvo como propósito central determinar cuál es el efecto de la orienta- ción al aprendizaje de las MIPYMES colombianas en su innovación.

Como resultado de esta investigación se puede señalar que al evidenciarse la relación ampliamente positiva y significativa entre la orientación al aprendizaje y la innovación de la empresa en cuanto a sus productos/servicios, procesos y gestión, se concluye y afirma que es altamente conveniente para las MIPYMES que sus directivos estimulen las siguientes prácticas a todo nivel de la empresa:

1) el planteamiento de nuevas ideas en cuanto a la forma de hacer el trabajo, la toma de iniciativas, la asunción de riesgos y el intercambio de conocimientos no solo al interior de la empresa sino con entidades como universidades y otras empresas;

2) la recompensa de las ideas innovadoras que funcionan, el abordaje constructivo de las fallas, y el cuestionamiento de las formas tradicionales de hacer las cosas; $y$

3) la revisión del entorno interno y externo de la empresa para encontrar nuevas perspectivas y posibilidades de desarrollo, la asunción positiva del cambio y de las nuevas ideas, porque todo ello redunda en incrementos significativos en el desempeño innovador de la empresa.

Los resultados de esta investigación son de gran importancia para los empresarios, porque muestra que si estimulan el aprendizaje organizacional, pueden recibir réditos en cuanto al nivel innovador de sus empresas, lo que a la postre genera un mejor rendimiento; a la academia, al sector gubernamental y a las entidades de apoyo al desarrollo empresarial, les confirma que deben orientar sus acciones hacia el cambio de paradigmas en los empresarios, para que estimulen la permanente actualización de sus colaboradores y demás recursos, porque esto es fundamental para mantener la competitividad en la actual sociedad del conocimiento.

Como limitación de la investigación se puede señalar que no se hicieron análisis para cada uno de los indicadores que conforman la orientación al aprendizaje organizacional sino sólo para la orientación al aprendizaje global; esto puede ser realizado en futuros trabajos para entender el impacto de cada uno de estos factores sobre la innovación de las MIPYMES. 


\section{Referencias}

Abbas, T. \& Alireza, M. (2011). Learning orientation, innovation and performance: evidence from small-sized business firms in Iran. EuropeanJournal of Social Sciences, 19 (1) (pp. 114-122).

Asociación Española de Contabilidad y Administración de Empresas (AECA). (1995). La innovación en la empresa: factor de supervivencia. Principios de organización y sistemas. Madrid, España: (AECA).

Argyris, C. \& Schön, S. (1978). Organizational learning: $A$ theory in action perspective. Reading, MA. USA: Addison-Wesley.

Baker, W. \& Sinkula, J. (1999). The synergistic effect of market orientation and learning orientation on organizational performance. Journal of the Academy of Marketing Science, 27 (4) (pp. 411-427).

Barney, J. (1991). Firm resources and sustained competitive advantage. Journal of Management, 17 (1) (pp. 99-120).

Bettis, R. \& Prahalad, C. (1995). The dominent logic: retrospective and extension. Strategic management journal, 16 (pp. 5-14).

Calantone, R., Cavusgil, T. \& Zhao, Y. (2002). Learning orientation, firm innovation capability, and firm performance. Industrial Marketing Management, 31 (pp. 515-524).

Camelo, C., Romero, P. y Valle, R. (2000). Relación entre el tipo y el grado de innovación y el rendimiento de la empresa. Economía Industrial, 333 (pp. 149-160).

Cardona, J. y Calderón, G. (2006). El impacto del aprendizaje en el rendimiento de las organizaciones. Cuadernos de Administración, Universidad Javeriana, 19 (32) (pp.11-43).

Cegarra, N. y Rodrigo-Moya, B. (2004). Desaprendizaje Individual: un paso previo a la creación del capital relacional. Cuadernos de Administración-Universidad Javeriana, 17 (27) (pp.11-32).

Comisión de las comunidades europeas. (2003). Comunicación de la Comisión al Consejo, al Parlamento Europeo, al Comité Económico y Social Europeo y al Comité de las Regiones (COM 2003, 112 final). Bruselas, Bélgica.
Conner, K. (1991). A historical comparison of resource-based theory and five schools of thought within industrial organization economics: Do we have a new theory of the firm? Journal of Management, 17 (1) (pp. 121-154).

Cyert, R. \& March, J. (1963).Teoría de las decisiones económicas en la empresas. México DF, México: Herrero Hermanos Sucesores, S.A Editores.

Damanpour, F.\& Gopalakrishnan, S. (2001). The dynamics of the adoption of products and process innovations in organizations. Journal of Management Studies, 38 (pp. 45-65).

Departamento Administrativo Nacional de Estadística (DANE). (2005). Censo general 2005. Bogotá, Colombia. Recuperado 14/10/2011 de http://www.dane.gov.co/censo/files/libroCens02005nacional.pdf

Day, G.S. (1991). Learning about markets (pp. 91-117). Recuperado 29/01/2013 de: http:// www.msi.org/publications/publication. cfm?pub=289

Day, G.S. (1994). Continuous learning about markets. Californian Management Review, 36 (pp. 9-31).

Dixon, N. (1992). Organizational learning: a review of the literature with implications for HRD professionals. Human Resource Development, 3 (pp. 29-49).

Drucker, P. (1985). La innovación y el empresario innovador. México DF, México: Edhasa.

Drucker, P. (1993). La sociedad post capitalista. Madrid, España: Editorial Deusto.

Formichella, M. (2005). Innovación del concepto de desarrollo y su relación con el desarrollo. Recuperado 30/11/2011 de: http://190.41.189.210/ oficinas/investigaciones/Evolucion $\% 20$ del\%2oConcepto\%20de $\% 2$ Innovacion $\% 20$ y\%20Desarrollo.pdf

García-Morales, V., Matías-Reche, F. \& VerdúJover, A. (2011) Influence of internal communication on technological proactivity, organizational learning, and organizational innovation in the pharmaceutical sector. Journal of Communication, 61, (pp. 150-177). 
Garzón, M. (2009). El aprendizaje organizacional en República Dominicana y Colombia. Pensamiento y Gestión, (26) (pp. 238-278).

Geus, A. (1988). Plannig learning. Harvard Business Review, 66 (pp. 70-74).

Goh, S. \& Richards, G. (1997). Benchmarking the learning capability of organizations. European Management Journal, 15 (pp. 575-583).

Grant, R. (1991). The resource base theory of competitive advantage. California Management Review, 33 (pp. 114-135).

Hage, J. (1999). Organizational innovation and organizational change. Annual Review of Sociology, 25 (pp. 597-622).

Hair, J., Anderson, R., Tatham, R. \& Black, W. (1999). Análisis Multivariante ( $5^{\underline{a}}$ ed.). Madrid, España: Prentice Hall.

Huber, G. (1991). Organizational learning: The contributing processes and the literatures. Organization Science, 2 (pp. 88-115).

Huber, G. (1998). Synergies between organizational learning and creativity \& innovation. Creativity \& Innovation Management, 7 (1) (p. 3).

Hurley, R. \& Hult, G. (1998). Innovation, market orientation, and organizational learning: an integration and empirical examination. Journal of Marketing, 62 (pp.42-54).

Jerez, P. (2001). Gestión de recursos humanos y aprendizaje: incidencia e implicaciones. Tesis doctoral. Universidad de Almería. Almería, España.

Jiménez, D. y Sanz, V. (2006). Innovación, aprendizaje organizacional y resultados empresariales: un estudio empírico. Cuadernos de Economía y Dirección de la Empresa, 29 (pp. 40-62).

Lado, A. y Wilson, M. (1994). Human resource systems and sustained competitive advantage: toward a conceptual integration. Journal of management, 18 (pp. 699-727).

Lippman, S. y Rumelt, R. (1982). Uncertain imitability: An analysis of interfirm differences in efficiency under competition. Bell Journal of Economics, 13 (pp. 418-438).

Ma, W., Zhu, G. \& Hou, Y. (2011). Learning orientation, process innovation, and firm performance in manufacturing industry. Advances in informationSciences and Service Sciences, 3 (11) (pp. 357-364).

Maldonado, G., Madrid, A., Martínez, M. y Aguilera, L. (2009). Los efectos de la innovación en el rendimiento de las MIPYMES de Aguascalientes: una evidencia empírica. Revista de economía, 27 (73) (pp.49-69). Recuperado el 5/03/2011 de http://www.revista.economia. uady.mx/2009/XXVI/73/02.pdf

Medina, C. y Espinoza, M. (1994). La innovación en las organizaciones modernas. Recuperado 12/06/2010 de: http://www.azc.uam.mx/ publicaciones/gestion/num5/doco6.htm

Miles, R. y Snow, C. (1978). Organizational strategy, structure and process. New York, USA: McGraw Hill.

Munuera, J. y Rodríguez, A. (2007). Estrategias de marketing: Un enfoque basado en el proceso de dirección. Madrid, España: ESIC Editor.

Nonaka, H. \& Takeuchi, H. (1995).The knowledgecreating company. How Japanese companies create the dynamics of innovation. New York, USA: Oxford University Press.

Nystrom, P. \& Starbuck, W. (1984). To avoid organizational crises, unlearn. Organizational Dynamics, 13 (pp. 53-65).

Organización para la Cooperación y Desarrollo Económicos (OECD), Oficina de Estadística de las Comunidades Europeas (EUROSTAT). (2005). Manual de Oslo. Oslo, Noruega: OECD.

Paños A., Ruiz, C., Sabater, R. y Ruiz, J. (2004). Modelo de análisis del aprendizaje organizacional. Efecto en la eficiencia organizacional e innovación. $2^{\circ}$ Congreso Soporte del Conocimiento con la Tecnología. Santander, España, 21 y 22 de mayo. (SOCOTE) (pp. 5-21).

Porac, J. \& Thomas, H. (1990). Taxonomic mental models in competitor definition. Academic of Management Review,15 (2) (pp. 224-40). 
Rogers, E. (1983). Diffussion and Innovation. New York, USA: Free Press.

Rumelt, R. (1984). Toward a Strategic Theory of the Firm. En: Lamb, R.B. (Ed.), Competitive Strategic Management. New Jersey, USA: Prentice-Hall (pp. 556-570).

Rumelt, R. (1987).Theory, Strategy, and entrepreneurship. En: Teece, D.J. (Ed.), The competitive challenge. Cambridge, USA: Ballinger Publishing (pp. 137-158).

Schumpeter, J. (1935). Teoría del desenvolvimiento económico. México DF, México: Fondo de Cultura Económica.

Senge, P. (1990). La quinta disciplina. El arte y la práctica de la organización abierta al aprendizaje. Barcelona, España: Granica.

Senge, P. (2000). La danza del cambio: los retos de sostener el impulso en organizaciones abiertas al aprendizaje. Barcelona, España: Ediciones Juan Granica S.A.
Sinkula, J., Baker, W. \& Noordewier, T. (1997). A framework for market-based organizational learning: Linking values, knowledge, and behavior. Journal of the Academy of Marketing Science, 25 (4) (pp. 305-319).

Slater, S. \& Narver, J. (1994). Market orientation isn't enough: build a learning organization. Cambridge, USA: Marketing Science Institute (pp. 94-103).

Slater, S. (1996). The challenge of sustaining competitive advantage. Industrial Marketing Management, 25 (pp. 79-86).

Thompson, V. (1965). Bureaucracy and innovation. Administrative Science Quarterly, 5 (pp.1-20).

Weerawardena, J., O`Cass, A., \& Julian, N. (2006). Does industry matter? Examining the role of industry structure and organizational learning in innovation and brand performance. Journal of Business Research, 59 (1) (pp. 37-45).

Wernerfelt, B. (1984). A Resource-based view of the firm. Strategic Management Journal, 5 (pp. 171-180). 